\title{
Over length quantification of the multiaxial mechanical properties of the ascending, descending and abdominal aorta using Digital Image Correlation
}

\author{
Juan A. Peña ${ }^{\text {a }}$ Victoria Corral ${ }^{b}$ Miguel A. Martínez ${ }^{\text {b,c }}$ Estefanía Peña b,c,* \\ ${ }^{a}$ Department of Management and Manufacturing Engineering. Faculty of Engineering and \\ Architecture. University of Zaragoza. Spain \\ ${ }^{\mathrm{b}}$ Aragón Institute of Engineering Research (I3A). Mechanical Engineering Department. University \\ of Zaragoza. Spain \\ ${ }^{\mathrm{c}}$ CIBER de Bioingeniería, Biomateriales y Nanomedicina (CIBER-BBN). Zaragoza. Spain
}

\begin{abstract}
In this paper, we hypothesize that the biaxial mechanical properties of the aorta may be dependent on arterial location. To demonstrate any possible position-related difference, our study analyzed and compared the biaxial mechanical properties of the ascending thoracic aorta, descending thoracic aorta and infrarenal abdominal aorta stemming from the same porcine subjects, and reported values of constitutive parameters for well-known strain energy functions, showing how these mechanical properties are affected by location along the aorta.

When comparing ascending thoracic aorta, descending thoracic aorta and infrarenal abdominal aorta, abdominal tissues were found to be stiffer and highly anisotropic. We found that the aorta changed from a more isotropic to a more anisotropic tissue and became progressively less compliant and stiffer with the distance to the heart. We observed substantial differences in the anisotropy parameter between aortic samples where abdominal samples were more anisotropic and nonlinear than the thoracic samples.

The phenomenological model was not able to capture the passive biaxial properties of each specific porcine aorta over a wide range of biaxial deformations, showing the best prediction root mean square error $\varepsilon=0.2621$ for ascending thoracic samples and, especially, the worst for the infrarenal abdominal samples $\varepsilon=0.3780$. The micro-structured model with Bingham orientation density function was able to better predict biaxial deformations ( $\varepsilon=0.1372$ for ascending thoracic aorta samples). The root mean square error of the micro-structural model and the micro-structured model with von Mises orientation density function were similar for all positions.
\end{abstract}

Key words: Biaxial testing, Ascending thoracic aorta, Descending thoracic aorta, Infrarenal abdominal aorta, Constitutive modeling, Digital Image Correlation. 


\section{Introduction}

Aorta mechanical properties vary along the aortic tree, generally agreeing that aortic stiffness increases with increasing distance from the heart (Guo and Kassab, 2003, Hang and Fung, 1995, Kim et al., 2013, Peña et al., 2015). Parameters such as aortic wall thickness, moisture content, and the location of the specimen along the aorta may influence the mechanical response. The fact that vessels differ so significantly in terms of anatomic characteristics is commonly attributed to the heterogeneity of blood flow within their territories during the arterial tree development phase (Dinardo et al., 2014). Determining the effect of position on gross mechanical properties has both experimental and clinical applications (García et al., 2011). Specifically, inflation (Lillie et al., 2012, Kim and Baek, 2011), planar biaxial (Zeinali-Davarani et al., 2013, Kamenskiy et al., 1998, Polzer et al., 2015) and uniaxial (Lally et al., 2004, Silver et al., 2003, Peña et al., 2015) testing are preferable in-vitro mechanical test protocols for vascular tissue. The aorta has been studied with great interest partially due to its large size, and partially due to its propensity to develop disease such as atherosclerotic process, dissection, and aneurysm. Here, animal models remain popular in clinical hypothesis testing, where specifically the pig aorta has a central role. In some cases, the validation of these models based only on uniaxial test data is inappropriate as biological and biomaterial membranes generally develop multiaxial stress states during real-life loading conditions. However, despite numerous studies on aortic properties, there is still little quantification of this effect by biaxial tests. Research to date has been limited by equibiaxial tests of descending aorta (Zeinali-Davarani et al., 2009, Peña et al., 2015), which found that distal thoracic samples are stiffer than proximal ones and anisotropy was more remarkable in lower thoracic aorta. There is limited data on the mechanical properties of ascending (Vorp et al., 2003, Guo and Kassab, 2004, Choudhury et al., 2009) and abdominal aorta (deGeest et al., 2004, Haskett et al., 2010, Kamenskiy et al., 1998). However, only Haskett et al. (2010) compared the ascending, descending and abdominal aorta biaxial mechanical properties.

To reproduce the mechanical behavior of these kinds of material, many constitutive models have been proposed for soft tissues (Fung, 1993, Holzapfel et al., 2000, Humphrey, 2002, Weiss et al., 1996). The preferred methodology to describe and reproduce its complex mechanical response is the definition of a strain energy function (SEF) from which the stress response is derived, see e.g. (Alastrué et al., 2009, Holzapfel et al., 2000, Zullinger et al., 2004, Gasser et al., 2006, Holzapfel and Ogden, 2010, Sokolis, 2010) and references therein. Although phenomenological models may reproduce the biomechanical properties of the vascular tissue, their material parameters lack a clear physical meaning. Moreover, these models are unreliable for predictions beyond the strain range used in parameter estimation (Polzer et al., 2015). Most these constitutive models simplify the micro-structure and assume fibers to be set out symmetrically relative to vessel axis, with a preferred orientation, yielding macroscopic orthotropic constitutive law for each layer (Rhodin, 1980). The response of fibers are typically assumed to be governed by exponential

\footnotetext{
* Corresponding author. Estefanía Peña. Mechanical Engineering Department. c/ Maria de Luna s/n 50018. Zaragoza. Spain. Tel.: +34 876555233; Fax: +34 976762578

Email address: fany@unizar.es (Estefanía Peña ).
} 
functions (Holzapfel et al., 2000, 2005). However, structurally-motivated material models may provide increased insights into the underlying mechanics and physics of arteries and could overcome this drawback (Weisbecker et al., 2015). The work by Gasser et al. (2006) included micro-structural information in the model by means of the assumption of a fiber orientation von Mises distribution function. Other models which assume the geometrical features of fibers follow a typical continuous distribution such as $\beta$-distribution with primarily planar array (Lanir, 1979, Hollander et al., 2011); more recently, models including fiber dispersion from a micro-structurally based approach have been proposed using axially symmetric von Mises orientation distribution function (ODF) around two preferred mean direction (Alastrué et al., 2009). More recently, Alastrué et al. (2010) proposed the use of the Bingham ODF for the incorporation of anisotropy in the micro-structurally based models. One of the main advantages of the Bingham ODF is the possibility of considering three different concentration parameters in three orthogonal directions of the space. These orientations can be easily correlated with the three main directions of a blood vessel: circumferential, radial and axial. Experimental studies have demonstrated that the non-symmetric Bingham ODF is more suitable to model aneurysms (Gasser et al., 2012), carotid (Sáez et al., 2016) and aorta (Polzer et al., 2015).

We hypothesized that the ascending aorta exhibits more isotropic biomechanical responses than descending and abdominal aorta with similar stiffness on circumferential and longitudinal directions to the artery. This behavior is more difficult to reproduce with the classical phenomenological strain energy functions used in the literature, and the micro-structurally based models fitted the experimental data very well. The aim of this study is to employ biaxial test methods to identify and quantify the effect of aortic region on the mechanical characteristics of the artery. Furthermore, our aim is (a) to investigate if changes to the mechanical properties are dependent on position and (b) to quantify these differences by estimation of the mechanical constitutive parameters by least-square fitting the recorded in-vitro biaxial test results. Since pigs are often used as models for cardiovascular studies, we designed the present study to analyze the mechanical behavior of porcine aortas.

\section{Experiments}

Porcine aortas $(\mathrm{n}=7)$ were harvested postmortem from approximately $3.5 \pm 0.6$ months-old female pigs, sacrificed for other studies that did not interfere with the aorta or the circulation system. The experiments on these swines were approved by the Ethical Committee for Animal Research of the University of Zaragoza and all procedures were carried out in accordance with the "Principles of Laboratory Animal Care" (86/609/EEC Norm). After artery harvesting and cleaning by removing excess connective tissue, they were kept frozen at $-20^{\circ} \mathrm{C}$ until testing. Once defrosted, samples were preserved in ion-free PSS $(0.9 \% \mathrm{NaCl})$ at $4\left[{ }^{\circ} \mathrm{C}\right]$ until preparation of testing samples was carried out. The aorta was subdivided into three parts: ascending thoracic (ATA), descending thoracic aorta (DTA) and infrarenal abdominal aorta (IAA). For this study, the analyzed specimens were obtained from the proximal region of each considered part (Peña et al., 2015). Samples were inspected for potential damage during slaughtering or harvesting. 
Square specimens, approximately $25 \times 25[\mathrm{~mm}]$, were cut from the ATA, DTA and IAA using a punch cutter and a scalpel. The specimens were prepared with their sides aligned in the circumferential and axial directions of the artery (Figure 1.a). A Mitutoyo Digimatic micrometer was used to measure the length, width and thickness of the samples using the average of three measurements for each sample.

\subsection{Mechanical testing}

Tests were carried out in a high precision drive system adapted for biological specimens Instron BioPuls $^{T M}$ low-force planar-biaxial testing system. Square specimens were mounted in the planar-biaxial machine by connecting four carriages by noddles clamps, immersed in a bath filled with PBS and maintained at $37^{\circ} \mathrm{C}$ by a heater-circulation. Noddles attaching the specimen were located as close as possible to the edges to minimize the edge effects on strain measurement (Figure 1.b). Load controlled tests were performed at three peak tension $(15,30$ and $60 \mathrm{kPa})$ ratios in circumferential and longitudinal directions $\left(P_{c}: P_{l}\right)$ corresponding to 0.5:0.5, 1:0.5, 0.5:1, 1:1, 2:1, 1:2, 2:2 tests at stress rates of approximately $2 \mathrm{kPa} / \mathrm{s}$ (Tong et al., 2011). An engineering stress (first Piola Kirchhoff stress tensor $\mathbf{P}$ ) of $30 \mathrm{kPa}$ served as a reference in the protocol. The experiment was stopped when tearing began at the noddles or when a final 2:2 protocol was applied. Samples were preconditioned through 10 loading cycles for each stress ratio, and the last cycle (tenth) was used for subsequent analysis. In turn, each measuring cycle comprises a loading and unloading curve. In order to obtain reproducible results, a preload of $5[\mathrm{mN}]$ to each specimen was applied.

[Fig. 1 about here.]

For the deformation measurements during biaxial testing, several randomized markers were placed on the surface of the vessel and the lengths between the two markers in each direction were measured by a Digital Image Correlation (DIC) Strain Master LaVision System equipped with two high performance digital cameras with a megapixel sensor $(2.5[\mu m] \pm 0.5 \%)$. The deformation gradient $\mathbf{F}$ was also measured optically by tracking the movements of markers (Figure 1.b).

Shear strains were computed and were small, and were not accounted for in the constitutive model. With negligible shear components and the incompressibility assumption, the first Piola Kirchhoff stresses (engineering stress) were determined as $P_{\theta \theta}=\frac{F_{\theta}}{t_{z} w_{z}}$ and $P_{z z}=\frac{F_{z}}{t_{\theta} w_{\theta}}$, where $t_{\theta, z}$ and $w_{\theta, z}$ are the initial thickness and width respectively. $F_{\theta, z}$ and $\lambda_{\theta, z}$ are the load registered by the biaxial machine and stretch measured by DIC technique in circumferential and longitudinal directions.

It would be helpful to measure the non-linearity and anisotropy of the samples in order to define certain levels of stress for which the corresponding stretches in the longitudinal and the circumferential directions can be compared at equibiaixal tension state. These values correspond to 30 and $60 \mathrm{kPa}$ of engineering stress. Anisotropy was analyzed by the difference in longitudinal and circumferential stretches divided by their average value (Kamenskiy et al., 1998). 


\subsection{Statistical analysis}

A statistical analysis was performed to study possible significant variations in the mechanical behavior of aorta along the position of the vessel. Therefore, the mechanical properties for the ATA, DTA and IAA were compared for both circumferential and longitudinal directions. Normal distribution of the variables was tested using Shapiro-Wilk test. The values were divided into three groups, corresponding to the ATA, DTA and IAA samples and all groups were compared by means of an independent one-tailed $t$-test. In cases of non-normal distribution, the MannWhitney test was used. $p<0.05$ was established to indicate statistical significance.

\section{Constitutive modeling}

Stress-stretch curves resulting from the tensile tests were used to fit several constitutive models. The fitted data were restricted to the elastic range, so data acquired after noticeable variations in the curve slope were neglected for the fitting procedure. Arterial tissue subject to loading exhibits strong nonlinearity, large strains and anisotropy, so the SEF is used to reproduce the mechanical behavior of the aortas. In order to generate constitutive mechanical parameters that could be used on computational simulations, each of the biaxial specimens tested was fitted to four constitutive models proposed in the literature.

\subsection{Phenomenological model}

The strong nonlinearity motivated the use of an exponential function for describing the strain energy stored in the collagen fibers that was previously proposed by Holzapfel et al. (2000). The main hypothesis was that each family of fibers represents the main direction of collagen bundles that are orientated in a helicoidal manner. Both families of fibers were assumed to have the same mechanical response and the anisotropy directions were assumed to be helically oriented at $\pm \theta$ degrees relative to circumferential direction (Holzapfel et al., 2000). Here, $\theta$ is treated as a phenomenological variable. The SEF proposed by the authors was

$$
\Psi=\mu\left(I_{1}-3\right)+\sum_{i=4,6}\left\{\frac{k_{1}}{2 k_{2}} \exp \left(k_{2}\left[I_{i}-1\right]^{2}\right]-1\right\},
$$

where

$$
I_{4}=\lambda_{\theta}^{2} \cos ^{2}(\theta)+\lambda_{z}^{2} \sin ^{2}(\theta), \quad I_{6}=\lambda_{\theta}^{2} \cos ^{2}(-\theta)+\lambda_{z}^{2} \sin ^{2}(-\theta) .
$$

In this equation $I_{1}$ represents the first invariant of the Cauchy-Green tensor (Spencer, 1971) characterizing the isotropic mechanical response of the elastin (Gundiah et al., 2009, Lillie et al., 2010). $\mu>0, k_{1}>0$ are stress-like parameters and $k_{2}>0$ is dimensionless. The SEF represents the strain energy stored in a composite material reinforced in two preferred directions represented by the invariants $I_{4}>1$ and $I_{6}>1$ where it has been assumed that the strain energy 
corresponding to the anisotropic terms only contributes to the global mechanical response of the tissue when stretched. A total of 4 elastic parameters $\left(\mu, k_{1}, k_{2}, \theta\right)$ should be fitted.

\subsection{Structural model}

The GOH model (Gasser et al., 2006) extended the model of Holzapfel et al. (2000) by the application of generalized structure tensor $\mathbf{H}=\kappa \mathbf{1}+(1-3 \kappa) \mathbf{M}_{0}$ (where $\mathbf{1}$ is the identity tensor and $\mathbf{M}_{0}=\mathbf{m}_{0} \otimes \mathbf{m}_{0}$ is a structure tensor defined using unit vector $\mathbf{m}_{0}$ specifying the mean orientation of fibers) and proposed a new constitutive model

$$
\left.\Psi=\mu\left(I_{1}-3\right)+\sum_{i=4,6}\left[\frac{k_{1}}{2 k_{2}}\left(\exp \left\{k_{2} \hat{E}_{i}\right]\right\}-1\right)\right]
$$

where

$$
\hat{E}_{i}=\kappa I_{1}+(1-3 \kappa) I_{i}-1 \quad i=4,6
$$

and $\kappa \in[0,1 / 3]$ is a dispersion parameter (the same for each collagen fiber family); when $\kappa=0$, the model is the same as the one published in Holzapfel et al. (2000), and when $\kappa=1 / 3$ it recovers an isotropic potential similar to that used in Demiray (1972). Note that the parameter $\kappa=\frac{1}{4} \int_{0}^{\pi} \rho \sin ^{3} \theta d \theta$ could have histological meaning due to the fully characterized distribution (Gasser et al., 2006). A total of 5 elastic parameters $\left(\mu, k_{1}, k_{2}, \kappa, \theta\right)$ should be fitted.

\subsection{Microfiber model}

Alastrué et al. (2010) proposed a microfiber model (microsphere-based model) to account for the dispersion of the collagen fibers around a preferential direction, overcoming the 1D limitation of previous characterization of the collagen fiber. Consistent with the constrained mixture approach (Humphrey and Rajagopal, 2003), we assume a free energy function of the form

$$
\Psi=\mu\left(I_{1}-3\right)+\Psi_{\text {coll }},
$$

where the subscripts coll refers to collagen fibers contribution. $\Psi_{\text {coll }}$ is defined as the sum of the contributions of each collagen family of fibrils as

$$
\Psi_{\text {coll }}=\sum_{j=1}^{N}\left[\Psi_{\text {coll }}\right]^{j}=\sum_{j=1}^{N}\left\langle n \rho \psi_{\text {coll }}\right\rangle^{j}=\sum_{j=1}^{N} \frac{1}{4 \pi} \int_{\mathbb{U}^{2}}\left(n \rho\left[\psi_{\text {coll }}\right]\right)^{j} d A
$$

where $N$ denotes the number of families of collagen fibers, $N=2$ according to the experimental results of orientation of collagen fibers (Holzapfel et al., 2000), and applying a discretization to the continuous orientation distribution on the unit sphere $\mathbb{U}^{2},\left[\Psi_{\text {coll }}\right]^{j}$ corresponds to the 
expression

$$
\left[\Psi_{\text {coll }}\right]^{j}=\sum_{i=1}^{m} n \rho\left(\mathbf{r}^{i} ;\left(\lambda_{\text {coll }}^{i}\right)\right.
$$

where $\mathbf{r}^{i}$ are the unit vectors associated with the discretization on the microsphere over the unit sphere $\mathbb{U}^{2}, m$ is the number of discrete orientation vectors (Alastrué et al., 2009), $\lambda_{\text {coll }}^{i}=\left\|\mathbf{F} \cdot \mathbf{r}^{i}\right\|$ the stretch in $\mathbf{r}^{i}$ direction and $\psi_{\text {coll }}^{i}\left(\lambda_{\text {coll }}^{i}\right)$ the strain energy function associated with $\mathbf{r}^{i}$ direction. Using Equations (6 and (7)), this results in

$$
\Psi_{\text {coll }}=\sum_{j=1}^{N} \sum_{i=1}^{m}\left(w^{i} n \rho\left[\psi_{\text {coll }}^{i}\right]\right)^{j}
$$

where $w_{i=1, \ldots . m}^{i}$ denotes related weighting factors and $\rho$ is the orientation density function (ODF) to take into account the fibrils dispersion (Alastrué et al., 2009).

The exponential-like strain energy function proposed by Holzapfel et al. (2000) was used to deal with the fiber response

$$
n \psi_{\text {coll }}^{i}\left(\lambda_{\text {coll }}^{i}\right)=\frac{c_{1 \text { coll }}}{2 c_{2 \text { coll }}}\left(e^{c_{2 \text { coll }}\left(\left(\lambda_{\text {coll }}^{i}\right)^{2}-1\right)^{2}}-1\right) \quad \text { if } \quad \lambda_{i} \geq 1 \quad \text { otherwise } \quad \psi_{\mathrm{f} i}\left(\lambda_{i}\right)=0
$$

since it is usually considered that collagen fibers only affect global mechanical behavior in tensile states, (Holzapfel et al., 2000). The affine kinematics define the collagen fiber stretch $\lambda_{\text {coll }}^{i}=\left\|\mathbf{t}^{i}\right\|$ in the fiber direction $\mathbf{r}^{i}$.

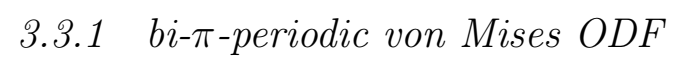

One of the ODF applied most frequently is $3 \mathrm{D}$ bi- $\pi$-periodic von Mises ODF for incorporation of anisotropy in a microsphere-based model with application to the modelling of the thoracic aorta (Alastrué et al., 2010). This function is expressed as

$$
\rho(\theta)=\rho_{1}(\theta)+\rho_{2}(\theta)
$$

where $\theta=\arccos (\mathbf{m} . \mathbf{r})$ is the so-called mismatch angle and $\mathbf{m}$ the preferred mean orientation of the collagen distribution, and

$$
\rho_{i}(\theta)=4 \sqrt{\frac{b}{2 \pi}} \frac{\exp (b[\cos (2 \theta)+1])}{\operatorname{erfi}(\sqrt{2 b})},
$$

where the positive concentration parameter $b$ constitutes a measure of the degree of anisotropy. Moreover, erfi $(x)=-i \operatorname{erf}(x)$ denotes the imaginary error function. Finally, $c_{1 \text { coll }}$ and $c_{2 \text { coll }}$ are stress dimensional and dimensionless material parameters respectively. A total of 5 elastic parameters $\left(\mu, k_{1}, k_{2}, \kappa, \theta\right)$ should be fitted. 


\subsubsection{Bingham $O D F$}

We also used the Bingham ODF (Bingham, 1974) initially proposed by Alastrué et al. (2010) for the incorporation of anisotropy in a microsphere-based model with application to the modelling of the thoracic aorta. One of the main advantages of the Bingham ODF is the possibility of considering three different concentration parameters in three orthogonal directions of the space. These orientations can be easily correlated with the three main directions of a blood vessel: circumferential, radial and axial. This function is expressed as

$$
\rho(\mathbf{r} ; \mathbf{Z}, \mathbf{Q}) \frac{d A}{4 \pi}=\left[F_{000}(\mathbf{Z})\right]^{-1} \exp \left(\operatorname{tr}\left(\mathbf{Z} \cdot \mathbf{Q}^{t} \cdot \mathbf{r} \cdot \mathbf{r}^{t} \cdot \mathbf{Q}\right)\right) \frac{d A}{4 \pi}
$$

where $\mathbf{Z}$ is a diagonal matrix with eigenvalues $\kappa_{1,2,3}, \mathbf{Q} \in \mathbb{Q}^{3}$ defining the orientation of the three principal orthogonal directions relative to the reference basis and

$$
F_{000}(\mathbf{Z})=\frac{1}{4 \pi} \int_{\mathbb{U}^{2}} \exp \left(\operatorname{tr}\left(\mathbf{Z} \cdot \mathbf{r} \cdot \mathbf{r}^{t}\right)\right) d A
$$

Thus, the probability of finding in a specific direction is controlled by the eigenvalues of Z, which might be interpreted as concentration parameters. Specifically, the difference between pairs of $\kappa_{1,2,3}-$ i.e., $\left[\kappa_{1}-\kappa_{2}\right],\left[\kappa_{1}-\kappa_{3}\right]$ and $\left[\kappa_{2}-\kappa_{3}\right]$ - determines the shape of the distribution over the surface of the unit sphere. Therefore, the value of one of these three parameters may be fixed to a constant value without reducing the versatility and different distributions of the family of fibers achieved for a constant value of $\kappa_{3}$ and varying values of $\kappa_{1}$ and $\kappa_{2}$. A total of 5 elastic parameters $\left(\mu, k_{1}, k_{2}, \kappa_{1}, \kappa_{2}\right)$ should be fitted.

\subsection{Parameter identification}

We fit the mechanical behavior of the tissue at physiological loads using the 2:1, 1:2, $2: 2$ protocols. The tissue was assumed as incompressible (Carew et al., 1968), i.e. $\operatorname{det}(\mathbf{F})=\lambda_{1} \lambda_{2} \lambda_{3}=$ 1 , where $\mathbf{F}$ represents the deformation gradient tensor and $\lambda_{i}, i=1,2,3$, the stretches in the principal directions. The fitting of the experimental data was developed by using a Nelder and Mead type minimization algorithm (Nelder and R.Mead, 1965) that is a heuristic search method that uses the concept of simplex by defining the objective function Eq.(14) using HyperFit software (www.hyperfit.wz.cz). In this function, $P_{\theta \theta}$ and $P_{z z}$ are the First PiolaKirchhoff (engineering) stress data obtained from the tests, $P_{\theta \theta}^{\Psi}=\frac{\partial \Psi_{\text {iso }}}{\partial \lambda_{\theta}}$ and $P^{\Psi}=\frac{\partial \Psi_{\text {iso }}}{\partial \lambda_{z}}$ are the First Piola-Kirchhoff (engineering) for the $i$ th point for a homogeneous pure biaxial state $\Psi$, and $n$ is the number of data points.

$$
\chi^{2}=\sum_{i=1}^{n}\left[\left(P_{\theta \theta}-P_{\theta \theta}^{\Psi}\right)_{i}^{2}+\left(P_{z z}-P_{z z}^{\Psi}\right)_{i}^{2}\right]
$$




\section{Results}

\subsection{Biaxial behavior}

A Tracking program (Strain Master LaVision System) was used for biaxial tensile stretch analysis. For each test, a user-defined grid was layered over the initial image of the sample and lengths between two markers in each direction were measured by a Digital Image Correlation (DIC), see Figure 2.a. A strain field plot derived from the grid displacement was used to assess alignment of experimental setup, strain uniformity on the center of the sample and neglected shear strains values (Figure 2). Measured shear strains on the center of the sample were found to be significantly lower than axial strains (by approximately two orders of magnitude) and were considered negligible (Figure 2.d).

[Fig. 2 about here.]

A representative biaxial mechanical response (Piola-Kirchhoff stress vs. stretch derived by DIC) are plotted in Figures 3, 4 and 5 for ATA, DTA and IAA for specimen VII, respectively. Tested specimens did not tear or exhibit signs of preliminary damage during testing. Generally, the force-controlled testing protocol allowed us to reach maximum stresses up to $60 \mathrm{kPa}$, but few sample stresses up to $120 \mathrm{kPa}$. The aorta revealed nonlinear, anisotropic and viscoelastic behavior (hysteresis formation) with a stiffer behavior and larger hysteresis in the abdominal part than in the ascending and descending aorta with stiffer behavior in the circumferential than in the longitudinal direction. For the ATA and DTA, the curves exhibit nearly elastic behavior with small hystereses. For the IAA, although the arterial specimens were of muscular type, hystereses were relatively small if we compare with other muscle arteries such as carotid (García et al., 2013). Softening between subsequent stress levels was clearly visible in the IAA (Figure 5.a) and is practically neglected for ATA and DTA wall, Figures 3.a and 4.a, respectively. The main softening occurred in the first cycle when the permanent stretch was increased. However, the elimination of this residual stretch resulted in similar material properties for each increased stress level. For the ATA, the permanent stretch is 1.0 showing a lower or null softening phenomena.

[Fig. 3 about here.]

A typical preconditioning behavior during equibiaxial loading at stress levels of 2:2 (60:60 kPa) is depicted in Figures 3.b, 4.b and 5.b for ATA, DTA and IAA, respectively, which was consistent in all tested specimens. The curves were stable and repeatable after a few preconditioning cycles, in particular three to four cycles were enough to precondition the ATA and DTA tissue and five to seven cycles for IAA tissue.

[Fig. 4 about here.]

Figures 3.c, 4.c and 5.c show a representative biaxial stress test behavior with different stress ratios between circumferential and longitudinal directions (0.5:0.5, 1:0.5, 0.5:1, 1:1, 2:1, 1:2, 
2:2) for ATA, DTA and IAA, respectively. The protocols $0.5: 1$ and 1:2 clearly depicted the anisotropy of the tissue where the circumferential data shows a compression stretch $(\lambda<1.0)$.

[Fig. 5 about here.]

A plot of axial stretch versus circumferential stretch at different ratios of specimen VII for ATA, DTA and IAA samples are presented in Figures 3.d, 4.d and 5.d for the tenth cycle. The permanent set commented above is clearly observed in the pictures, showing high values on the longitudinal direction. The stiffer behavior of the distal samples is also depicted. For ATA samples the maximum stretch are 1.1 and 1.12 for circumferential and longitudinal directions for 2:2 protocol, the equivalent values for the DTA are 1.17 and 1.38, and for the IAA were 1.095 and 1.165. These results demonstrated that ascending thoracic aorta shows a quasi-isotropic response.

Finally, Figures 3.e and .f, 4.e and .f and 5.e and .f show the mean and standard deviation of the equibiaxial 2:2 experimental data for whole samples in circumferential (e) and (f) longitudinal directions. For all positions, the standard deviation is lower for the circumferential direction than longitudinal one. It is notable that longitudinal direction is stiffer than the circumferential one for specimens III, V and VI for ATA.

The average biaxial Cauchy stress-stretch behavior obtained from all considered aortic specimens $(\mathrm{n}=7)$ was computed and plotted in Figure 6. A stiffer response in circumferential directions is evident in the IAA region compared to the ATA $(p<0.01)$ and DTA region $(p<0.05)$, particularly at higher stretches, see Table 1, where stretches at $30 \mathrm{kPa}$ and $60 \mathrm{kPa}$ for all curves and the anisotropy parameter for ATA, DTA and IAA samples are summarized. In contrast, the longitudinal tests show similar stress-stretch curves for all locations. The statistical analysis revealed significant differences between the mechanical behavior from ATA, DTA to IAA samples in circumferential directions $(p<0.05)$. In the case of the tensile tests in longitudinal direction, there are no significant differences between all locations ( $p=0.73$ for ATA to DTA, $p=0.32$ for ATA to IAA and $p=0.49$ for DTA to IAA).

[Fig. 6 about here.]

[Table 1 about here.]

\subsection{Constitutive parameters}

Due to little softening effect, only the three last protocols $(2: 1,1: 2,2: 2)$ were considered for the fitting procedure. The experimental data were fitted using the proposed SEFs following the procedure explained in Section 3.4. The results of the fitting to the SEFs for ATA, DTA and IAA samples are shown in Tables 2, 3 and 4, respectively. Our results on the descriptive capacity of SEFs models indicated that the worst fitting was with the HGO SEF for all positions, especially for the IAA samples, showing a mean RMSE of $\varepsilon>0.25(\varepsilon=0.2621, \varepsilon=0.2668$ and $\varepsilon=0.3780$ for ATA, DTA and IAA samples, respectively). On the contrary, the best fitting was with the 
micro-structured model with Bingham ODF function showing a mean RMSE of $\varepsilon=0.1372$, $\varepsilon=0.1767$ and $\varepsilon=0.3166$ for ATA, DTA and IAA samples, respectively. The RMSE of the GOH model and the micro-structured model with von Mises ODF function were similar for all positions. It is remarkable that all the considered SEF present a questionable fitting for IAA with $\varepsilon>0.3$. Regarding the predictive capacity of the material models, the fitted material constants using only the equibiaxial test (2:2) demonstrated a good predictive result for the biaxial tests $(2: 1$ and $1: 2)$, data not shown, with a "predictive" error, $\varepsilon_{\text {error }}<10 \%$, (relative difference between the best fit of the equibiaxial test and the $\varepsilon$ for the reproduction of the biaxial curves) for the Bingham micro-structured model only.

[Table 2 about here.]

[Table 3 about here.]

[Table 4 about here.]

A statistically-relevant difference between the ascending, descending and abdominal aortas was found for the constitutive parameters for parameter $\mu$ for HGO and GOH models $(p<0.01)$, and between ascending with descending and abdominal aortas, $(p<0.05)$ but not for descending with abdominal ones for the micro-structured model with von Mises ODF function. In general, the constitutive parameters $\mu$ decrease with position. For $k_{1}$ parameter, there is no a clear tendency and depends on the model. There is a statistical difference between ascending and descending aortas (micro-structured models, $p<0.05$ ), between ascending and descending with abdominal aortas (HGO $p<0.05)$ and ascending with abdominal aortas(GOH $p<0.05$ ). The $k_{2}$ parameter presents a significant decrease with all positions for HGO and GOH models $(p<0.05)$ and an increase for micro-structured models with a significance between ascending and descending aortas $(p<0.05)$. No position dependency for the $\theta$ parameter was found for all models and positions $(p>0.41)$. The dispersion parameter $\kappa$ for the GOH model decreases with position, with a significance between ascending and descending positions $(p<0.05)$. On the contrary, the concentration parameter b of von Mises ODF increases significantly with position $(p<0.01)$. Note that $\kappa$ is a dispersion parameter where $\kappa=0$ means no dispersion (Gasser et al., 2006) and $b$ is a concentration parameter where $b=0$ represents maximum dispersion with random/isotropic fiber distribution (Alastrué et al., 2009). Finally, the constitutive parameters $\kappa_{1}$ and $\kappa_{2}$ of the Bingham ODF function increase statistically with position for ATA, DTA and IAA samples $(p<0.05)$. Note that $\kappa_{1}$ and $\kappa_{2}$ describe the degree of anisotropy and represent the concentration of fiber orientations around specific principal axis 1 (circumferential) or 2 (longitudinal).

\section{Discussion}

In this paper, we hypothesize that the biaxial mechanical properties of the aorta may be dependent on arterial location. To demonstrate any possible position-related difference, our study analyzed and compared the biaxial mechanical properties of the ascending thoracic aorta (ATA), 
descending thoracic aorta (DTA) and infrarenal abdominal aorta (IAA) stemming from the same porcine subjects and reported values of constitutive parameters for well-known SEFs, showing how these mechanical properties are affected by location along the aorta.

Our results showed that the excised porcine aorta is non-linear and anisotropic with the longitudinal direction being more compliant. When comparing ascending thoracic aorta (ATA), descending thoracic aorta (DTA) and infrarenal abdominal aorta (IAA), IAA tissues were found to be stiffer and highly anisotropic. We found that the aorta changed from a more isotropic to a more anisotropic tissue and became progressively less compliant and stiffer with distance to the heart. We observed substantial differences in the anisotropy parameter between ATA, DTA and IAA samples where IAA samples were more anisotropic and nonlinear than the ATA and DTA samples. Similar results were presented by Choudhury et al. (2009) which did not find significant anisotropy for ascending thoracic aorta. However, Kamenskiy et al. (1998), Polzer et al. (2015) and Zeinali-Davarani et al. (2013) found anisotropy properties for descending aorta, and deGeest et al. (2004), Haskett et al. (2010) and Weisbecker et al. (2012) for abdominal aorta. The statistical analysis revealed significant differences between the mechanical behavior of ATA, DTA and IAA locations in circumferential direction, but not in the longitudinal one. This observation is consistent with the results of Peña et al. (2015) which found significant differences between the mechanical behavior of proximal and distal locations along the thoracic aorta in circumferential directions, but not in the longitudinal one. A hypothesis of this finding is due to the fact the degree of fiber alignment was also found to increase along the length of the aorta but to be primarily in circumferential direction (Schriefl et al., 2012). Similarly, Zeinali-Davarani et al. (2015) demonstrated that the degree of collagen undulation was a factor leading to the difference in mechanical properties along the thoracic aorta.

Hysteresis were relatively small, i.e. the specimens behaved nearly elastically. Moreover, preconditioning was finished typically after only a few loading-unloading cycles. In particular three to four cycles were enough to precondition the ATA and DTA tissue and five to seven cycles for more muscular IAA tissue. There is no equivalent analysis in the literature. Only Weisbecker et al. (2012) analyze the effct of the preconditioning on the mechanical properties of human descending thoracic and abdominal aortas. However, this analysis was only at the mechanical parameters of the model proposed by the authors and there is no proof analysis of the curves. Our finding is in accordance with Weisbecker et al. (2012), that found more softening and preconditioning effect on abdominal aortas than descending thoracic ones. This effect is totally removed under collagen digestion and increased with elastase treatment on the media from the human thoracic aorta, Weisbecker et al. (2013). This effect would be increased on the abdominal aorta where preconditioning on healthy tissue is higher than on descending thoracic aorta, and could help to understand the effect of abdominal aneurysm on mechanical properties of abdominal aorta (Pierce et al., 2015).

Several constitutive models for arteries have been proposed in the literature and overviews are provided on some reviews (Humphrey, 1995, Holzapfel and Ogden, 2010). In this work, we study a series of constitutive models, some with a phenomenological approach and others which are micro-structural and physically-oriented, describing the features of the arterial wall with greater accuracy. We aim to examine the advantages and limitations of phenomenological versus more 
micro-structural oriented approaches. In this direction, the stress-stretch curves obtained were fitted with a hyperelastic anisotropic models which are well-known in the literature, (Holzapfel et al., 2000, Gasser et al., 2006, Alastrué et al., 2009, 2010), providing a reasonable approximation of the experimental data. The phenomenological model (Holzapfel et al., 2000) was not able to capture the passive biaxial properties of each specific porcine aorta over a wide range of biaxial deformations, showing the best prediction mean RMSE $\varepsilon=0.2621$ for ATA samples and, especially, the worst for the IAA samples $\varepsilon=0.3780$. The micro-structural model with Bingham ODF function was able to better predict the biaxial deformations $(\varepsilon=0.1372$ for ATA samples). The RMSE of the GOH model and the micro-structured model with von Mises ODF function were similar for all positions.

However, despite the error results it is worth mentioning the fundamental fact of physically motivated results. The phenomenological model (Holzapfel et al., 2000) predicted a mean fiber orientation of $\theta_{\mathrm{ATA}}=55.4371^{\circ}, \theta_{\mathrm{DTA}}=72.3025^{\circ}$ and $\theta_{\text {dist }}=79.77^{\circ}$ without dispersion. This fiber orientation does not match with the experimental observations in Schriefl et al. (2012) where collagen fibers were observed mainly along $\theta \approx 45^{\circ}$ of circumferential direction with high dispersion for both descending and abdominal locations. Furthermore, with regards to the measure of the fiber dispersion, $\kappa \approx 0.3$ for GOH model and $b \approx 0$, the micro-structured model with von Mises ODF function and mean fiber orientation are in keeping with the dispersed distribution obtained in the experimental results of Schriefl et al. (2012). In accordance with Schriefl et al. (2012), the micro-structural model with Bingham ODF function showed $\kappa_{1}-$ $\kappa_{2} \approx 0$ meaning high dispersion around the circumferential direction. However, in spite of the good fitting results of GOH model, only the micro-stuctured materials models showed a "predictive" error, $\varepsilon_{\text {error }}<10 \%$.Regarding the parameter estimation analysis, the larger the number of parameters, the more flexible and better fitting (in terms of residual error), as would be expected. However, too many parameters not only increase the complexity of the model (Zeinali-Davarani et al., 2009) but also increment the disadvantages of ill-posed problems. In this regard we agree that the main goal in constitutive models should be to include physically motivated aspects and, as much as possible, to feed these models with experimental data obtained from histological analysis, polarized light microscopy (Schriefl et al., 2012) or other quantitative experimental techniques (Rezakhaniha et al., 2012).

Limitations to our study need to be acknowledged before closing. Regarding the experimental analysis, a small number of tissue samples $(n=7)$ were investigated, hence a meaningful correlation between biomechanical properties of the different arterial tissues with related geometrical parameters could not be quantified. All mechanical properties were reported under the assumption of homogeneity that clearly is not the case for arteries. Porcine tissues make a convenient test model. However, biaxial material and structural differences were observed between the human and porcine tissues (Martin et al., 2011). Aortas were kept frozen at $-20^{\circ} \mathrm{C}$ until testing. No significant difference was found between the elastic modulus of porcine aortic tissue before and after freezing at $-20^{\circ} \mathrm{C}$ and was unaffected by storage time (O'Leary et al., 2014). However, freezing of the artery specimens kills smooth muscle cells, and the mechanical properties measured in the present study is those of deactivated tissues without smooth muscle tone even though the experiments were performed in $37^{\circ} \mathrm{C}$. We assumed that the smooth muscle in the segments was in resting (noncontracted) state. The hysteresis would be relatively small 
in this study due to we used frozen specimens whose smooth muscle activity is completely lost. Finally, the noddles clamps induced additional tissue damage, so the maximum load before rupture is lower than uniaxial tests, and the data obtained with biaxial testing are lower for describing the essential nonlinearity and anisotropy of these materials under physiologic loads.

With regards to material fitting, only data in the range used to fit the model is theoretically predictable. The anisotropy of the elastin, which has been shown to play a non-negligible mechanical role (Zeinali-Davarani et al., 2009, Zou and Zhang, 2009, 2011)), was not considered. The waviness of collagen fibers was not included by a probability density function for the recruitment stretch at which the fiber starts to bear load (Weisbecker et al., 2015). Our model captures only the passive mechanical behavior aortic tissues. The present formulation does not take into account viscoelastic effects, since after preconditioning most tissues demonstrated relatively narrow hysteresis. Finally, we assume the individual tissues as incompressible materials (Carew et al., 1968). However, there is data in the literature that reveal that ovine aortic tissue is highly compressible, showing an effective Poisson's ratio of 0.44 (Nolan and McGarry, 2016). We do not have enough information to check this hypothesis.

Current study underscores the need for further studies on the layer-specific mechanical tests and micro-stuctured constitutive models that take into account experimental data obtained from histological analysis with the help of advanced microscopic imaging (Weisbecker et al., 2015).

\section{Acknowledgments}

The authors are indebted to C. Marzo and A. Sanesteban for their valuable contributions to the experimental tests. Furthermore, financial support for this research was provided by the Spanish Ministry of Economy and Competitiveness through research project DPI2016-76630C2-1-R and DPI2014-51763-REDT; the Department of Industry and Innovation (Government of Aragon) through the research group Grant T88 (European Social Fund) and the Instituto de Salud Carlos III (ISCIII) through the CIBER initiative. The work was performed by the ICTS "NANBIOSIS" specifically by the Tissue \& Scaffold Characterization Unit (U13), of the CIBER in Bioengineering, Biomaterials \& Nanomedicne (CIBER-BBN at the University of Zaragoza).

\section{Conflict of interest}

None of the authors of this work has conflict of interest with other people and organizations. The funders had no role in study design, data collection and analysis, decision to publish, or preparation of the manuscript. 


\section{References}

Alastrué, V., Martínez, M. A., Doblaré, M., Menzel, A., 2009. Anisotropic micro-sphere-based finite elasticity applied to blood vessel modelling. J Mech Phys Solids 57, 178-203.

Alastrué, V., Saez, P., Martínez, M. A., Doblaré, M., 2010. On the use of bingham statistical distribution in microsphere-based constitutive models for arterial tissue. Mech Res Commun 37, 700-706.

Bingham, C., 1974. An antipodally summetric distribution on the sphere. Ann Stat 2, 12011225.

Carew, T. E., Vaishnav, R. N., Patel, D. J., 1968. Compressibility of the arterial wall. Circ Res $23,61-86$.

Choudhury, N., Bouchot, O., Rouleau, L., Tremblay, D., Cartier, R., Butany, J., Mongrain, R., Leask, R. L., 2009. Local mechanical and structural properties of healthy and diseased human ascending aorta tissue. Cardiovasc Pathol 18, 83-91.

deGeest, J. P. V., Sacks, M. S., Vorp, D. A., 2004. Age dependency of the biaxial biomechanical behavior of human abdominal aorta. ASME J Biomech Eng 126, 815.

Demiray, H., 1972. A note on the elasticity of soft biological tissues. J Biomech 5, 309-311.

Dinardo, C., Venturini, G., Zhou, E. H., Watanabe, I. S., Campos, L. C., Dariolli, R., da MottaLeal-Filho, J. M., Carvalho, V. M., Cardozo, K. H., Krieger, J. E., Alencar, A. M., AC, A. C. P., 2014. Variation of mechanical properties and quantitative proteomics of VSMC along the arterial tree. Am J Physiol Heart Circ Physiol 306, H505-H516.

Fung, Y. C., 1993. Biomechanics. Mechanical properties of living tissues. Springer-Verlag.

García, A., Martínez, M. A., Peña, E., 2013. Determination and Modeling of the Inelasticity Over the Length of the Porcine Carotid Artery. ASME J Biomech Eng 135, 031004-1.

García, A., Peña, E., Laborda, A., Lostalé, F., de Gregorio, M. A., Doblaré, M., Martínez, M. A., 2011. Experimental study and constitutive modelling of the passive mechanical properties of the porcine carotid artery and its relation to histological analysis. Implications in animal cardiovascular device trials. Med Eng Phys 33, 665-676.

Gasser, T. C., Gallinetti, S., Xing, X., Forsell, C., Swedenborg, J., Roy, J., 2012. Spatial orientation of collagen fibers in the abdominal aortic aneurysm's wall and its relation to wall mechanics. Acta Biomat 8, 3091-3103.

Gasser, T. C., Ogden, R. W., Holzapfel, G. A., 2006. Hyperelastic modelling of arterial layers with distributed collagen fibre orientations. J R Soc Interface 3, 15-35.

Gundiah, N., Ratcliffe, M. B., Pruitt, L. A., 2009. The biomechanics of arterial elastin. J Mech Behav Biomed Mater 2, 288-296.

Guo, X., Kassab, G. S., 2003. Variation of mechanical properties along the length of the aorta in C57bl/6 mice. Am J Physiol Heart Circ Physiol 285, H2614-H2622.

Guo, X., Kassab, G. S., 2004. Distribution of stress and strain along the porcine aorta and coronary arterial tree. Am J Physiol Heart Circ Physiol 286, H2361-H2368.

Hang, H. C., Fung, Y. C., 1995. Longitudinal strain of canine and porcine aortas. J Biomech 28, 637-641.

Haskett, D., Johnson, G., Zhou, A., Utzinger, U., Geest, J. V., 2010. Microstructural and biomechanical alterations of the human aorta as a function of age and location. Biomechan Model Mechanobiol 9, 725-736.

Hollander, Y., Durban, D., Lanir, Y., 2011. Experimentally validated microstructural 3D con- 
stitutive model of coronary arterial media. J Biomech Eng 133, 031007.

Holzapfel, G. A., Gasser, C. T., Sommer, G., Regitnig, P., 2005. Determination of the layerspecific mechanical properties of human coronary arteries with non-atherosclerotic intimal thickening, and related constitutive modelling. Am J Physiol Heart Circ Physiol 289, H2048H2058.

Holzapfel, G. A., Gasser, T. C., Ogden, R. W., 2000. A new constitutive framework for arterial wall mechanics and a comparative study of material models. J Elasticity $61,1-48$.

Holzapfel, G. A., Ogden, R. W., 2010. Constitutive modelling of arteries. Phil Trans R Soc A 466, 1551-1597.

Humphrey, J. D., 1995. Mechanics of the arterial wall: Review and directions. Crit Rev Biomed Eng 23, 1-162.

Humphrey, J. D., 2002. Continuum biomechanics of soft biological tissues. Proc R Soc Lond A $175,1-44$.

Humphrey, J. D., Rajagopal, K. R., 2003. A constrained mixture model for arterial adaptations to a sustained step change in blood flow. Biomech Model Mechanobiol 2, 109-126.

Kamenskiy, A. V., Dzenis, Y. A., Jaffar-Kazmi, S. A., Pemberton, M. A., Pipinos, I. I., Phillips, N. Y., Herber, K., Woodford, T., Bowen, R. E., Lomneth, C. S., MacTaggart, J. N., 1998. Biaxial mechanical properties of the human thoracic and abdominal aorta, common carotid, subclavian, renal and common iliac arteries. Biomech Model Mechanobiol 13, 1341-1359.

Kim, J., Baek, S., 2011. Circumferential variations of mechanical behavior of the porcine thoracic aorta during inflation test. J Biomech 44, 1941-1947.

Kim, J., Hong, J.-W., Baek, S., 2013. Longitudinal differences in the mechanical properties of the thoracic aorta depend on circumferential regions. J Biomed Mater Res Part A 101, 1525-1529.

Lally, C., Reid, A. J., Prendergast, P. J., 2004. Elastic behavior of porcine coronary artery tissue under uniaxial and equibiaxial tension. Ann Biomed Eng 32, 1355-1364.

Lanir, Y., 1979. A structural theory for the homogeneous biaxial stress-strain relationship in flat collageneous tissues. J Biomech 12, 423-436.

Lillie, M. A., Shadwick, R. E., Gosline, J. M., 2010. Mechanical anisotropy of inflated elastic tissue from the pig aorta. J Biomech 43, 2070-2078.

Lillie, M. A., Shadwick, R. E., Gosline, J. M., 2012. Contribution of elastin and collagen to the inflation response of the pig thoracic aorta: Assessing elastin's role in mechanical homeostasis. J Biomech 45, 2133-2141.

Martin, C., Pham, T., Sun, W., 2011. Significant differences in the material properties between aged human and porcine aortic tissues. Eur J Cardiothorac Surg 40, 28-34.

Nelder, J. A., R.Mead, 1965. A simplex method for function minimization. Computer Journal 7, 308-313.

Nolan, D. R., McGarry, J. P., 2016. On the Compressibility of Arterial Tissue. Ann Biomed Eng 44, 993-1007.

O'Leary, S. A., Doyle, B. J., McGloughlin, T. M., 2014. The impact of long term freezing on the mechanical properties of porcine aortic tissue. J Mech Behav Biomed 37, 165-173.

Peña, J. A., Martínez, M. A., Peña, E., 2015. Layer-specific residual deformations and uniaxial and biaxial mechanical properties of thoracic porcine aorta. J Mech Behav Biomed 50, 55-69.

Pierce, D. M., Maier, F., Weisbecker, H., Viertler, C., Verbrugghe, P., Famaey, N., Fourneau, I., Herijgers, P., Holzapfel, G. A., 2015. Human thoracic and abdominal aortic aneurysmal 
tissues: Damage experiments, statistical analysis and constitutive modeling. J Mech Behav Biomed 41, 92-107.

Polzer, S., Gasser, T. C., Novak, K., Mana, V., Tichy, M., Skacel, P., Bursa, J., 2015. Structurebased constitutive model can accurately predict planar biaxial properties of aortic wall tissue. Acta Biomater 14, 133-145.

Rezakhaniha, R., Agianniotis, A., Schrauwen, J. T. C., Griffa, A., Sage, D., Bouten, C. V. C., van de Vosse, F. N., Unser, M., Stergiopulos, N., 2012. Experimental investigation of collagen waviness and orientation in the arterial adventitia using confocal laser scanning microscopy. Biomech Model Mechanobiol 11, 461-473.

Rhodin, J. A. G., 1980. Architecture of the vessel wall, Handbook of Physiology, The Cardiovascular System, vol. 2. American Physiological Society, Bethesda, MD.

Schriefl, A., Zeindlinger, G., Pierce, D., Regitnig, P., Holzapfel, G., 2012. Determination of the layer-specific distributed collagen fiber orientations in human thoracic and abdominal aortas and common iliac arteries. J R Soc Interface 9, 1275-1286.

Sáez, P., García, A., Peña, E., Gasser, T. C., Martínez, M. A., 2016. Microstructural quantification of collagen fiber orientations and its integration in constitutive modeling of the porcine carotid artery. Acta Biomat 33, 183-193.

Silver, F. H., Snowhill, P. B., Foran, D. J., 2003. Mechanical behavior of vessel wall: A comparative study of aorta, vena cava, and carotid artery. Ann Biomed Eng 31, 793-803.

Sokolis, D. P., 2010. A passive strain-energy function for elastic and muscular arteries: correlation of material parameters with histological data. Med Biol Eng Comput 48, 507-518.

Spencer, A. J. M., 1971. Theory of Invariants. In: Continuum Physics. Academic Press, New York, pp. 239-253.

Tong, J., Cohnert, T., Regitnig, P., Holzapfel, G., 2011. Effects of age on the elastic properties of the intraluminal thrombus and the thrombus-covered wall in abdominal aortic aneurysms: Biaxial extension behaviour and material modelling. Eur J Vas Endovas Surg 42, 207-219.

Vorp, D. A., Schiro, B. J., Ehrlich, M. P., Juvonen, T. S., Ergin, M. A., Griffith, B. P., 2003. Effect of aneurysm on the tensile strength and biomechanical behavior of the ascending thoracic aorta. Ann Thorac Surg 75, 1210-1214.

Weisbecker, H., Pierce, D. M., Regitnig, P., Holzapfel, G. A., 2012. Layer-specific damage experiments and modeling of human thoracic and abdominal aortas with non-atherosclerotic intimal thickening. J Mech Behav Biomed 12, 93-106.

Weisbecker, H., Pierce, D. M., Regitnig, P., Holzapfel, G. A., 2013. The role of elastin and collagen in the softening behavior of the human thoracic aortic media. J Biomech 46, 18591865.

Weisbecker, H., Unterberger, M. J., Holzapfel, G. A., 2015. Constitutive modelling of arteries considering fibre recruitment and three-dimensional fibre distribution. J R Soc Interface 12, 20150111.

Weiss, J. A., Maker, B. N., S.Govindjee, 1996. Finite element implementation of incompressible, transversely isotropic hyperelasticity. Comput Methods Appl Mech Engrg 135, 107-128.

Zeinali-Davarani, S., Choi, J., Baek, S., 2009. On parameter estimation for biaxial mechanical behavior of arteries. J Biomech 42, 524-530.

Zeinali-Davarani, S., Chow, M.-J., Turcotte, R. L., Zhang, Y., 2013. Characterization of Biaxial Mechanical Behavior of Porcine Aorta under Gradual Elastin Degradation. Ann Biomed Eng 41, 1528-1538. 
Zeinali-Davarani, S., Wang, Y., Chow, M.-J., Turcotte, R. L., Zhang, Y., 2015. Contribution of Collagen Fiber Undulation to Regional Biomechanical Properties Along Porcine Thoracic Aorta. ASME J Biomech Eng 137, 051001-1.

Zou, Y., Zhang, Y., 2009. An experimental and theoretical study on the anisotropy of elastin network. Ann Biomed Eng 37, 1572-1583.

Zou, Y., Zhang, Y., 2011. The orthotropic viscoelastic behavior of aortic elastin. Biomech Model Mechanbiol 10, 613-625.

Zullinger, M., Rachev, A., Stergiopulos, N., 2004. A constitutive formulation of arterial mechanics including vascular smooth muscle tone. Am J Physiol Heart Circ Physiol 287, H1335H1343. 


\begin{tabular}{ccccccc}
\hline \hline Specimen & $\lambda_{\theta}^{30}$ & $\lambda_{z}^{30}$ & $A^{30}$ & $\lambda_{\theta}^{60}$ & $\lambda_{z}^{60}$ & $A^{60}$ \\
\hline ATA & $1.075 \pm 0.030$ & $1.103 \pm 0.047$ & $0.036 \pm 0.020$ & $1.119 \pm 0.044$ & $1.144 \pm 0.052$ & $0.050 \pm 0.024$ \\
DTA & $1.064 \pm 0.024$ & $1.110 \pm 0.048$ & $0.043 \pm 0.040$ & $1.089 \pm 0.028$ & $1.136 \pm 0.053$ & $0.046 \pm 0.047$ \\
IAA & $1.030 \pm 0.036$ & $1.127 \pm 0.045$ & $0.104 \pm 0.036$ & $1.048 \pm 0.042$ & $1.15 \pm 0.048$ & $0.112 \pm 0.041$ \\
\hline
\end{tabular}

Table 1

Circumferential $\left(\lambda_{\theta}^{30}, \lambda_{\theta}^{60}\right)$ and longitudinal $\left(\lambda_{z}^{30}, \lambda_{z}^{60}\right)$ stretches and anisotropy measurements corresponding to $30 \mathrm{kPa}$ and $60 \mathrm{kPa}$ during equibiaxial test $(2: 2)$, respectively. Anisotropy $\left(A^{30}\right.$ and $\left.A^{60}\right)$ was analyzed by the difference in longitudinal and circumferential stretches at each stress level $(30 \mathrm{kPa}$ and $60 \mathrm{kPa}$ respectively) divided by their average value. Values are presented as Average \pm Standard Deviation 


\begin{tabular}{|c|c|c|c|c|c|c|c|}
\hline HGO Model & & & & & & & \\
\hline Specimen & $\mu$ & $k_{1}$ & $k_{2}$ & $\theta$ & $R^{2}$ & $\varepsilon$ & \\
\hline$I$ & 0.0491 & 0.0014 & 13.3111 & 51.45 & 0.5797 & 0.3473 & \\
\hline$I I$ & 0.0649 & 0.0086 & 14.2597 & 86.52 & 0.7170 & 0.2628 & \\
\hline$I I I$ & 0.0751 & 0.0010 & 42.4897 & 90 & 0.6957 & 0.2547 & \\
\hline$I V$ & 0.0496 & 0.0291 & 14.3696 & 70.21 & 0.7777 & 0.2492 & \\
\hline$V$ & 0.0947 & 0.0013 & 36.6047 & 0.88 & 0.7915 & 0.2385 & \\
\hline$V I$ & 0.0885 & 0.0001 & 76.9745 & 89 & 0.7496 & 0.2491 & \\
\hline$V I I$ & 0.1042 & 0.0156 & 15.4189 & 0 & 0.8172 & 0.2334 & \\
\hline Mean & 0.0751 & 0.0081 & 30.4897 & 55.4371 & 0.7326 & 0.2621 & \\
\hline$S D$ & 0.0217 & 0.0108 & 23.7617 & 39.9397 & 0.0795 & 0.03882 & \\
\hline \multicolumn{8}{|l|}{ GOH Model } \\
\hline Specimen & $\mu$ & $k_{1}$ & $k_{2}$ & $\kappa$ & $\theta$ & $R^{2}$ & $\varepsilon$ \\
\hline$I$ & 0.0102 & 0.1597 & 38.4863 & 0.3168 & 0 & 0.6964 & 0.2435 \\
\hline$I I$ & 0.0208 & 0.6296 & 0.0010 & 0.3244 & 0 & 0.7483 & 0.2345 \\
\hline$I I I$ & 0.0298 & 0.5284 & 0.0010 & 0.3333 & 0 & 0.7233 & 0.2342 \\
\hline$I V$ & 0.0391 & 0.0010 & 189.9870 & 0.1145 & 82.09 & 0.8303 & 0.2283 \\
\hline$V$ & 0.0300 & 1.1446 & 0.0010 & 0.3304 & 13.20 & 0.8455 & 0.1882 \\
\hline$V I$ & 0.0313 & 0.6674 & 0.001 & 0.3333 & 90 & 0.8310 & 0.2003 \\
\hline$V I I$ & 0.0293 & 1.8021 & 0.3175 & 0.333 & 0 & 0.8161 & 0.1844 \\
\hline Mean & 0.0268 & 0.5217 & 38.0795 & 0.2921 & 30.8816 & 0.7791 & 0.2215 \\
\hline$S D$ & 0.0100 & 0.4057 & 75.9946 & 0.0872 & 43.1067 & 0.0642 & 0.0219 \\
\hline \multicolumn{8}{|c|}{ Microfiber Von Mises Model } \\
\hline Specimen & $\mu$ & $k_{1}$ & $k_{2}$ & $b$ & $\theta$ & $R^{2}$ & $\varepsilon$ \\
\hline$I$ & 0.0011 & 0.1100 & 0.1569 & 0 & 88.80 & 0.7654 & 0.2797 \\
\hline$I I$ & 0.0011 & 0.2312 & 0.001 & 0 & 87.24 & 0.8412 & 0.2348 \\
\hline$I I I$ & 0.0014 & 0.2084 & 0.3632 & 0 & 55.34 & 0.7982 & 0.2453 \\
\hline$I V$ & 0.0010 & 0.2779 & 0.9266 & 0 & 61.28 & 0.8646 & 0.2040 \\
\hline$V$ & 0.001 & 0.2768 & 2.9591 & 0.1774 & 0 & 0.9722 & 0.0947 \\
\hline$V I$ & 0.001 & 0.2701 & 0.7339 & 0 & 25.31 & 0.8977 & 0.1711 \\
\hline$V I I$ & 0.001 & 0.3324 & 0.6507 & 0 & 17.86 & 0.6392 & 0.3164 \\
\hline Mean & 0.0010 & 0.2438 & 0.8273 & 0.0253 & 47.9757 & 0.8255 & 0.2208 \\
\hline \multicolumn{8}{|l|}{$S D$} \\
\hline \multicolumn{8}{|c|}{ Microfiber Bingham model } \\
\hline Specimen & $\mu$ & $k_{1}$ & $k_{2}$ & $\kappa_{1}$ & $\kappa_{2}$ & $R^{2}$ & $\varepsilon$ \\
\hline I & 0.0010 & 0.04600 & 1.7403 & 8.6308 & 7.6538 & 0.9227 & 0.1598 \\
\hline$I I$ & 0.0013 & 0.1874 & 2.1677 & 0.9100 & 0.0319 & 0.9801 & 0.0789 \\
\hline$I I I$ & 0.0462 & 0.1100 & 0.0123 & 0 & 0 & 0.8683 & 0.2081 \\
\hline$I V$ & 0.0010 & 0.2826 & 0.001 & 0.5428 & 0 & 0.9555 & 0.1223 \\
\hline$V$ & 0.0012 & 0.2993 & 0.0011 & 0.2373 & 0 & 0.9743 & 0.09248 \\
\hline$V I$ & 0.0010 & 0.2509 & 0.7153 & 0 & 0 & 0.9118 & 0.1620 \\
\hline$V I I$ & 0.0305 & 0.2653 & 0.0056 & 1.1022 & 0 & 0.9182 & 0.1676 \\
\hline Mean & 0.0086 & 0.1960 & 0.7729 & 1.7201 & 1.2809 & 0.9354 & 0.1372 \\
\hline$S D$ & 0.0184 & 0.1011 & 0.9648 & 3.4034 & 3.1220 & 0.04274 & 0.0485 \\
\hline
\end{tabular}

Table 2

Material constants obtained for the ascending thoracic aorta (ATA) curves. Constants $\mu$ and $k_{1}$ in MPa, $\theta$ in degrees, $k_{2}, \rho, \kappa, \mathrm{b}, \kappa_{1}$ and $\kappa_{2}$ are dimensionless. 


\begin{tabular}{|c|c|c|c|c|c|c|c|}
\hline HGO Mode & & & & & & & \\
\hline Specimen & $\mu$ & $k_{1}$ & $k_{2}$ & $\theta$ & $R^{2}$ & $\varepsilon$ & \\
\hline$I$ & 0.0531 & 0.0051 & 16.4048 & 63.31 & 0.9382 & 0.1458 & \\
\hline$I I$ & 0.001 & 0.0175 & 1.7351 & 80.15 & 0.6592 & 0.3459 & \\
\hline$I I I$ & 0.0145 & 0.0117 & 3.0238 & 78.11 & 0.8329 & 0.2626 & \\
\hline$I V$ & 0.0314 & 0.01363 & 8.5495 & 72.82 & 0.7947 & 0.3048 & \\
\hline$V$ & 0.0263 & 0.0134 & 11.2069 & 79.88 & 0.8750 & 0.2486 & \\
\hline$V I$ & 0.0435 & 0.0037 & 93.9559 & 57.43 & 0.8295 & 0.2316 & \\
\hline VIIa & 0.0129 & 0.0038 & 6.7227 & 67.58 & 0.6670 & 0.4269 & \\
\hline$V I I b$ & 0.0010 & 0.0150 & 2.7861 & 79.14 & 0.2827 & 0.1686 & \\
\hline Mean & 0.0229 & 0.0104 & 18.0481 & 72.3025 & 0.7349 & 0.2668 & \\
\hline$S D$ & 0.01908 & 0.0054 & 31.0638 & 8.6528 & 0.2063 & 0.0919 & \\
\hline \multicolumn{8}{|l|}{ GOH Model } \\
\hline Specimen & $\mu$ & $k_{1}$ & $k_{2}$ & $\kappa$ & $\theta$ & $R^{2}$ & $\varepsilon$ \\
\hline$I$ & 0.0262 & 0.0117 & 26.06 & 0.1125 & 59.83 & 0.9415 & 0.1422 \\
\hline$I I$ & 0.0054 & 0.0654 & 7.6320 & 0.2726 & 20 & 0.6858 & 0.3431 \\
\hline$I I I$ & 0.0078 & 0.0947 & 4.6290 & 0.2885 & 1.5 & 0.8680 & 0.1701 \\
\hline$I V$ & 0.0146 & 0.1906 & 11.1502 & 0.2848 & 17.69 & 0.8200 & 0.2875 \\
\hline$V$ & 0.001 & 0.3552 & 0.0014 & 0.2742 & 74 & 0.8840 & 0.2256 \\
\hline$V I$ & 0.0210 & 0.0862 & 660.0371 & 0.2712 & 0.0 & 0.8494 & 0.2139 \\
\hline VIIa & 0.0064 & 0.0258 & 18.9642 & 0.2531 & 0.0 & 0.6668 & 0.4268 \\
\hline$V I I b$ & 0.0025 & 0.1035 & 6.7001 & 0.2895 & 0.0 & 0.8558 & 0.2663 \\
\hline Mean & 0.0106 & 0.1166 & 91.8967 & 0.2558 & 21.6275 & 0.8214 & 0.2594 \\
\hline$S D$ & 0.0090 & 0.1107 & 229.7130 & 0.0590 & 29.3472 & 0.0961 & 0.0931 \\
\hline \multicolumn{8}{|c|}{ Microfiber Von Mises Model } \\
\hline Specimen & $\mu$ & $k_{1}$ & $k_{2}$ & $b$ & $\theta$ & $R^{2}$ & $\varepsilon$ \\
\hline$I$ & 0.0021 & 0.1742 & 0.0010 & 0.2155 & 48.31 & 0.9362 & 0.0995 \\
\hline$I I$ & 0.0015 & 0.0511 & 0.0016 & 1.2059 & 42.66 & 0.7827 & 0.3385 \\
\hline$I I I$ & 0.0016 & 0.0630 & 0.5198 & 0.5402 & 12.60 & 0.9670 & 0.1305 \\
\hline$I V$ & 0.0016 & 0.1224 & 0.8446 & 0.6631 & 11.34 & 0.9048 & 0.2371 \\
\hline$V$ & 0.0010 & 0.1033 & 3.0416 & 0.8904 & 41.82 & 0.9516 & 0.1806 \\
\hline$V I$ & 0.0028 & 0.1318 & 13.0384 & 0.6237 & 38.01 & 0.96281 & 0.1192 \\
\hline VIIa & 0.0037 & 0.0426 & 0.0012 & 0.9761 & 40.56 & 0.6912 & 0.4821 \\
\hline$V I I b$ & 0.0010 & 0.0565 & 0.0011 & 0.8537 & 12.83 & 0.8936 & 0.2918 \\
\hline Mean & 0.0019 & 0.0931 & 2.1811 & 0.74607 & 33.6471 & 0.8862 & 0.2349 \\
\hline$S D$ & 0.0009 & 0.0472 & 4.5067 & 0.3029 & 15.0599 & 0.0987 & 0.1312 \\
\hline \multicolumn{8}{|c|}{ Microfiber Bingham model } \\
\hline Specimen & $\mu$ & $k_{1}$ & $k_{2}$ & $\kappa_{1}$ & $\kappa_{2}$ & $R^{2}$ & $\varepsilon$ \\
\hline$I$ & 0.0342 & 0.0604 & 7.2772 & 0.5158 & 0.0005 & 0.9821 & 0.0812 \\
\hline$I I$ & 0.0010 & 0.0288 & 1.2392 & 8.7994 & 6.6073 & 0.8435 & 0.1734 \\
\hline$I I I$ & 0.001 & 0.0503 & 0.5371 & 2.4282 & 1.4002 & 0.9696 & 0.1308 \\
\hline$I V$ & 0.0011 & 0.1153 & 0.8676 & 1.6350 & 0.3303 & 0.9013 & 0.2220 \\
\hline$V$ & 0.001 & 0.1129 & 2.8115 & 1.2928 & 0.0 & 0.9508 & 0.1806 \\
\hline$V I$ & 0.0010 & 0.1365 & 11.6631 & 5.4277 & 4.5481 & 0.9568 & 0.1230 \\
\hline VIIa & 0.001 & 0.0361 & 1.7047 & 1.9521 & 0.0 & 0.7633 & 0.3207 \\
\hline$V I I b$ & 0.0011 & 0.0198 & 2.2892 & 14.9106 & 12.7635 & 0.9503 & 0.1823 \\
\hline Mean & 0.0051 & 0.0700 & 3.5487 & 4.6202 & 3.2062 & 0.9147 & 0.1767 \\
\hline$S D$ & 0.0117 & 0.0449 & 3.9072 & 4.9679 & 4.5837 & 0.0756 & 0.0726 \\
\hline
\end{tabular}

Table 3

Material constants obtained for the descending thoracic aorta (DTA) curves. Constants $\mu$ and $k_{1}$ in MPa, $\theta$ in degrees, $k_{2}, \rho, \kappa, \mathrm{b}, \kappa_{1}$ and $\kappa_{2}$ are dimensionless. 


\begin{tabular}{|c|c|c|c|c|c|c|c|}
\hline HGO Model & & & & & & & \\
\hline Specimen & $\mu$ & $k_{1}$ & $k_{2}$ & $\theta$ & $R^{2}$ & $\varepsilon$ & \\
\hline$I$ & 0.0156 & 0.0553 & 12.6284 & 73.78 & 0.6439 & 0.3681 & \\
\hline$I I$ & 0.0010 & 0.0342 & 7.5036 & 89 & 0.7871 & 0.4180 & \\
\hline$I I I$ & 0.0001 & 0.0449 & 2.8768 & 78.43 & 0.8488 & 0.2345 & \\
\hline$I V$ & 0.0009 & 0.0333 & 7.6543 & 89 & 0.7907 & 0.4165 & \\
\hline$V$ & 0.0019 & 0.1343 & 8.8924 & 77.54 & 0.3365 & 0.5150 & \\
\hline$V I$ & 0.0001 & 0.0226 & 0.001 & 84.53 & 0.4185 & 0.3456 & \\
\hline$V I I a$ & 0.0016 & 0.0610 & 7.3177 & 78.30 & 0.8289 & 0.2995 & \\
\hline$V I I b$ & 0.0129 & 0.0038 & 6.7220 & 67.58 & 0.6670 & 0.4269 & \\
\hline Mean & 0.0042 & 0.0486 & 6.6995 & 79.77 & 0.6651 & 0.3780 & \\
\hline$S D$ & 0.0062 & 0.0390 & 3.8057 & 7.4301 & 0.1929 & 0.0863 & \\
\hline \multicolumn{8}{|l|}{ GOH Model } \\
\hline Specimen & $\mu$ & $k_{1}$ & $k_{2}$ & $\kappa$ & $\theta$ & $R^{2}$ & $\varepsilon$ \\
\hline$I$ & 0.0038 & 0.4072 & 0.0140 & 0.2169 & 61.07 & 0.7709 & 0.2920 \\
\hline$I I$ & 0.001 & 0.7722 & 0.001 & 0.3185 & 0 & 0.8596 & 0.3431 \\
\hline$I I I$ & 0.0014 & 0.1873 & 0.0010 & 0.2066 & 65.54 & 0.8765 & 0.2095 \\
\hline$I V$ & 0.0009 & 0.7729 & 0.001 & 0.3183 & 7.02 & 0.8597 & 0.3423 \\
\hline$V$ & 0.0361 & 0.1163 & 0.0001 & 0 & 0 & 0.3857 & 0.5058 \\
\hline$V I$ & 0.0009 & 0.0212 & 0.0009 & 0.0019 & 83.98 & 0.5734 & 0.3557 \\
\hline VIIa & 0.0023 & 0.4724 & 0.001 & 0.2499 & 63.04 & 0.8559 & 0.2788 \\
\hline$V I I b$ & 0.0064 & 0.0258 & 18.9679 & 0.2531 & 0 & 0.6680 & 0.4213 \\
\hline Mean & 0.0066 & 0.3469 & 2.3733 & 0.1956 & 35.0812 & 0.7312 & 0.3435 \\
\hline$S D$ & 0.0120 & 0.3092 & 6.7052 & 0.1269 & 6.3621 & 0.1770 & 0.0905 \\
\hline \multicolumn{8}{|c|}{ Microfiber Von Mises Model } \\
\hline Specimen & $\mu$ & $k_{1}$ & $k_{2}$ & $b$ & $\theta$ & $R^{2}$ & $\varepsilon$ \\
\hline$I$ & 0.0011 & 0.1027 & 10.0179 & 5.7195 & 61.38 & 0.8699 & 0.2147 \\
\hline$I I$ & 0.0010 & 0.1166 & 5.4504 & 1.774 & 21.50 & 0.8174 & 0.3785 \\
\hline$I I I$ & 0.0010 & 0.0549 & 3.3565 & 8.0044 & 67.83 & 0.8977 & 0.1802 \\
\hline$I V$ & 0.0011 & 0.0365 & 11.9555 & 8.7805 & 73.32 & 0.9338 & 0.2337 \\
\hline$V$ & 0.0014 & 0.2168 & 3.1256 & 6.5461 & 65.80 & 0.5419 & 0.4488 \\
\hline$V I$ & 0.0013 & 0.0487 & 0.0046 & 0.6089 & 31.62 & 0.5765 & 0.3785 \\
\hline$V I I a$ & 0.0010 & 0.1806 & 0.0010 & 0.8553 & 0 & 0.7130 & 0.3537 \\
\hline$V I I b$ & 0.0010 & 0.0362 & 1.6980 & 1.2902 & 39.00 & 0.7442 & 0.4195 \\
\hline Mean & 0.0011 & 0.0991 & 4.4511 & 4.1973 & 45.0562 & 0.7618 & 0.3259 \\
\hline$S D$ & 0.0001 & 0.0688 & 4.44672 & 3.4164 & 26.2309 & 0.1455 & 0.1016 \\
\hline \multicolumn{8}{|c|}{ Microfiber Bingham model } \\
\hline Specimen & $\mu$ & $k_{1}$ & $k_{2}$ & $\kappa_{1}$ & $\kappa_{2}$ & $R^{2}$ & $\varepsilon$ \\
\hline$I$ & 0.001 & 0.1307 & 0.0010 & 14.1782 & 11.5926 & 0.6954 & 0.3485 \\
\hline$I I$ & 0.0052 & 0.0708 & 5.7774 & 12.9714 & 9.6194 & 0.8141 & 0.3118 \\
\hline$I I I$ & 0.0010 & 0.0707 & 0.6437 & 14.998 & 12.36 & 0.7104 & 0.2702 \\
\hline$I V$ & 0.0018 & 0.0729 & 5.4166 & 14.9998 & 11.7925 & 0.8085 & 0.2409 \\
\hline$V$ & 0.0010 & 0.2687 & 4.4931 & 9.9572 & 6.6388 & 0.5063 & 0.4104 \\
\hline$V I$ & 0.0005 & 0.02591 & 0.0028 & 16.0015 & 15.0984 & 0.5843 & 0.2722 \\
\hline VIIa & 0.001 & 0.0751 & 5.9988 & 14.9213 & 13.0486 & 0.7760 & 0.2794 \\
\hline$V I I b$ & 0.0010 & 0.0361 & 3.4276 & 1.7047 & 1.9520 & 0.7663 & 0.3997 \\
\hline Mean & 0.0011 & 0.0991 & 4.4511 & 4.1973 & 45.0562 & 0.7618 & 0.3259 \\
\hline$S D$ & 0.0001 & 0.0688 & 4.4467 & 3.4164 & 26.2309 & 0.1455 & 0.1016 \\
\hline
\end{tabular}

Table 4

Material constants obtained for the infrarenal abdominal aorta (IAA) curves. Constants $\mu$ and $k_{1}$ in MPa, $\theta$ in degrees, $k_{2}, \rho, \kappa, \mathrm{b}, \kappa_{1}$ and $\kappa_{2}$ are dimensionless. 


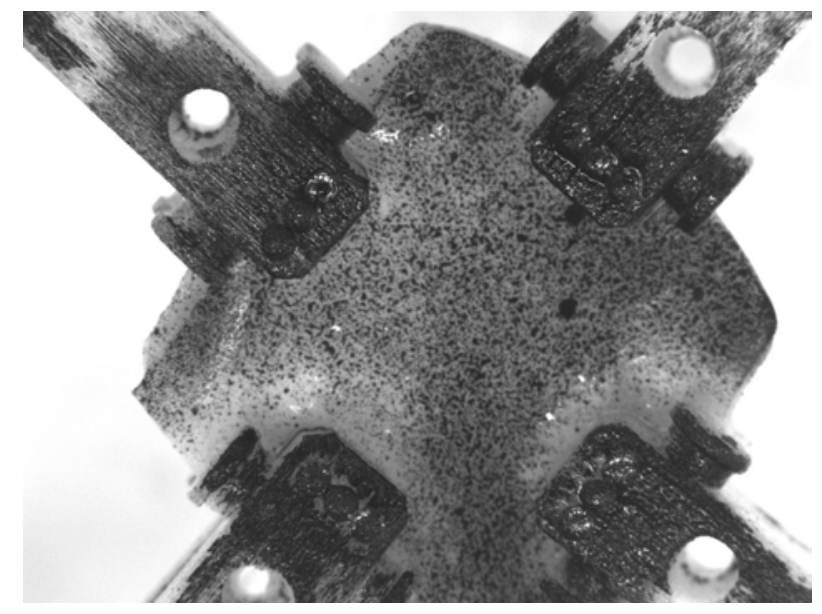

(a) Biaxial setup

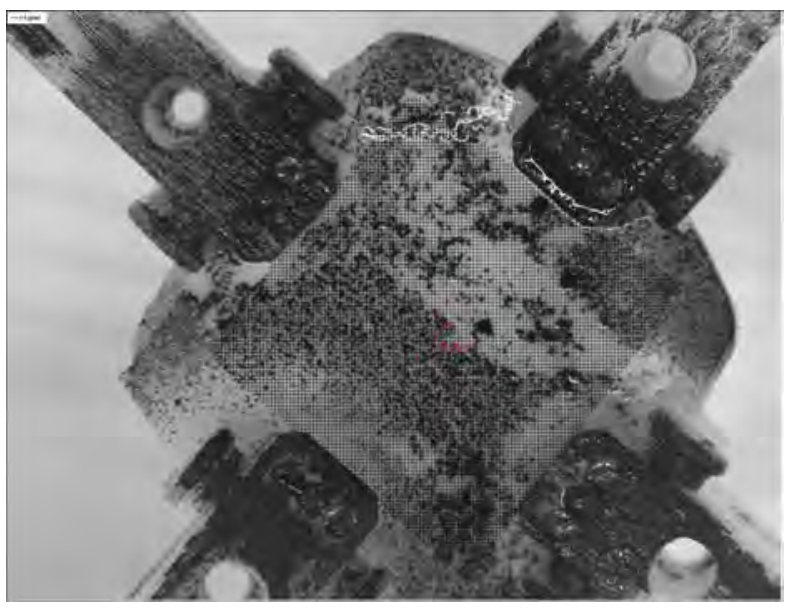

(b) Biaxial DIC grid

Fig. 1. (a) Representative image of the specimen mounted in a biaxial tensile testing device after the application of a pre-load. (b) Application of a grid on the surface of the vessel and the lengths between the two markers in each direction were measured by a Digital Image Correlation (DIC) Strain Master LaVision System. 


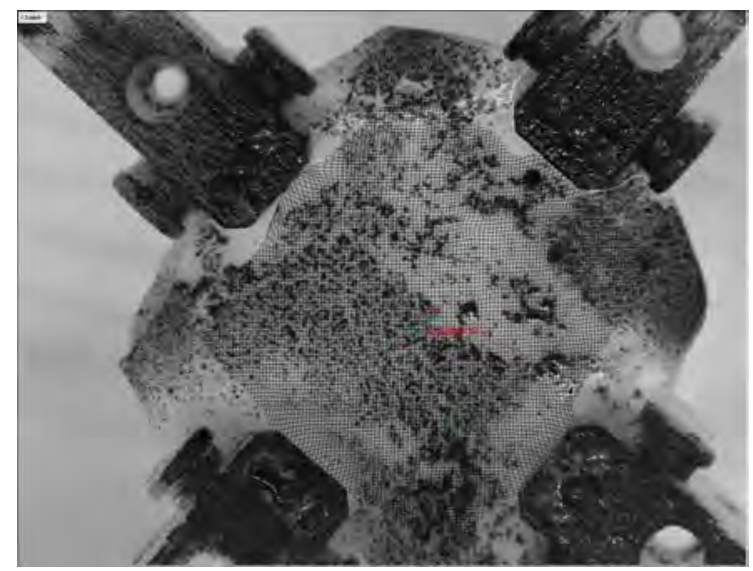

(a) Deformed grid

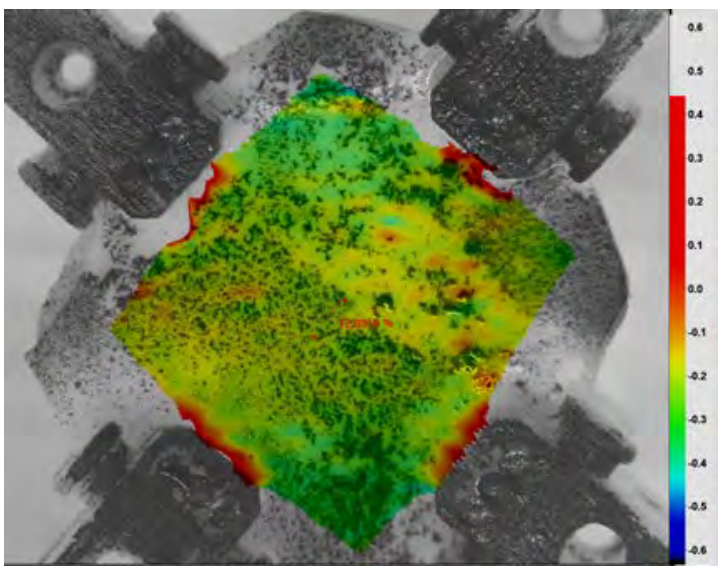

(c) Circumferential strain field

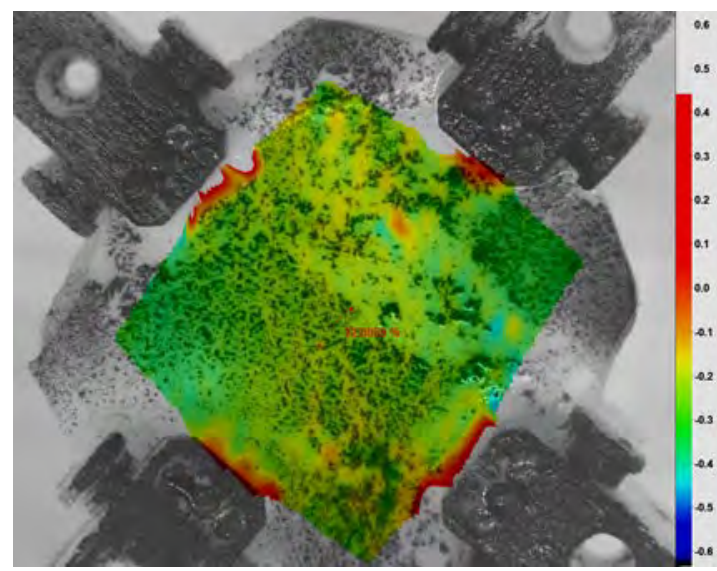

(b) Longitudinal strain field

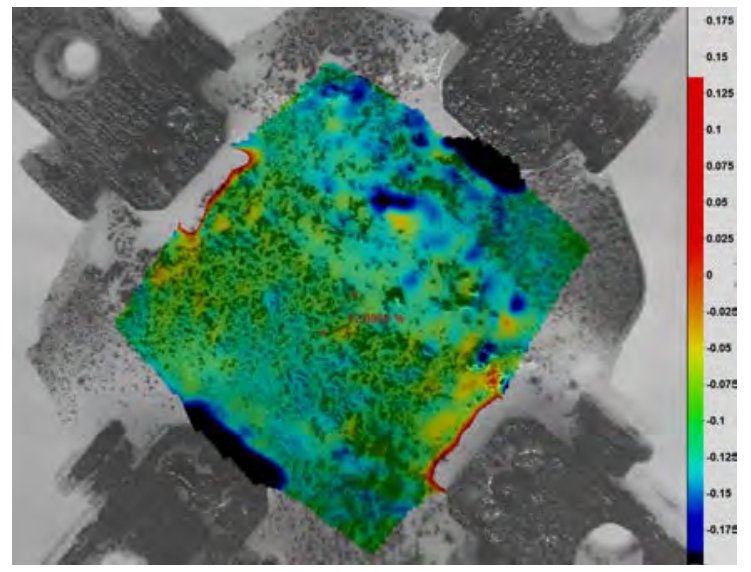

(d) Shear strain field

Fig. 2. Representative (specimen XX) equibiaxial analysis (2:2) showing (a) the deformed grid, the (b) circumferential, (c) longitudinal and (d) shear strain map at the end of the experiment. 


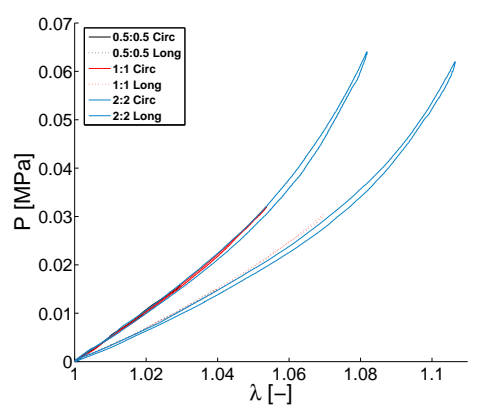

(a) Equibiaxial cycles

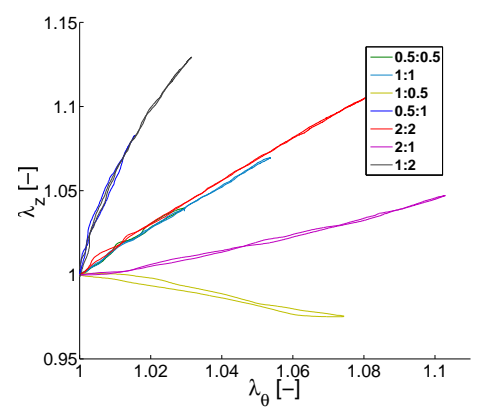

(d) Stretch

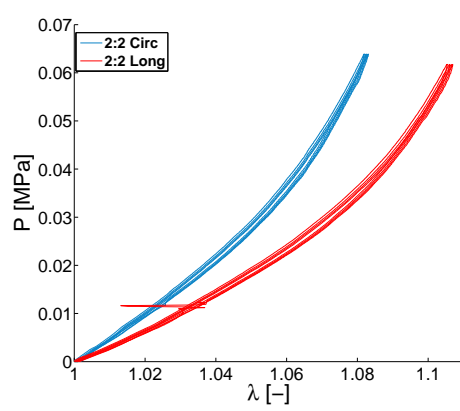

(b) Preconditioning 2:2

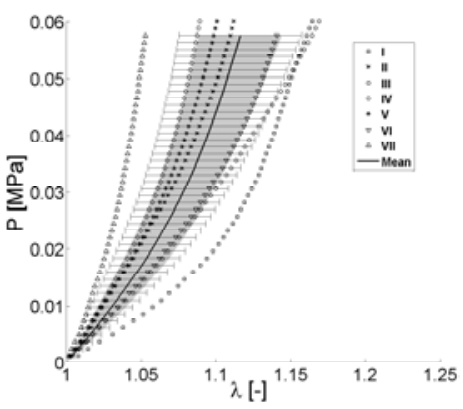

(e) Circumferential

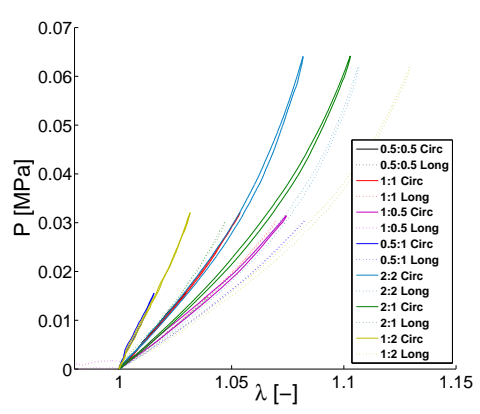

(c) Biaxial

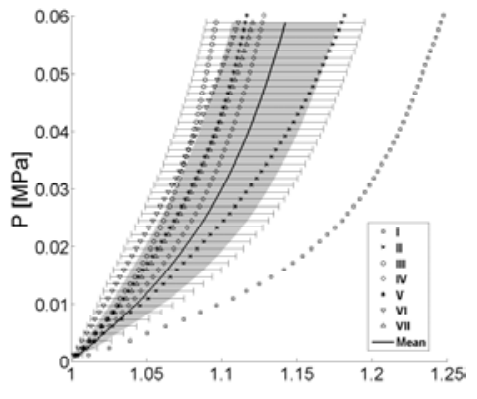

(f) Longitudinal

Fig. 3. Representative (specimen VIIa) Piola-Kirchhoff stress vs. stretch behavior for the porcine ascending thoracic aorta (ATA) specimens: (a) typical preconditioned equibiaxial properties at subsequently increased stress levels ranging from 15 to $60[\mathrm{kPa}]$; (b) representative preconditioning behavior in terms of loading-unloading cycles at $60[\mathrm{kPa}] ;$ (c) typical biaxial behavior at different ratios 0.5:0.5, 1:0.5, 0.5:1, 1:1, 2:1, 1:2, $2: 2$; (d) typical axial stretch versus circumferential stretch at different ratios; (e) whole equibiaxial 2:2 experimental data samples in circumferential and (f) longitudinal directions. Colored areas represent $\mathrm{p}>0: 1$ and $\mathrm{p}<0: 9$ of the equibiaxial 2:2 experimental data. 


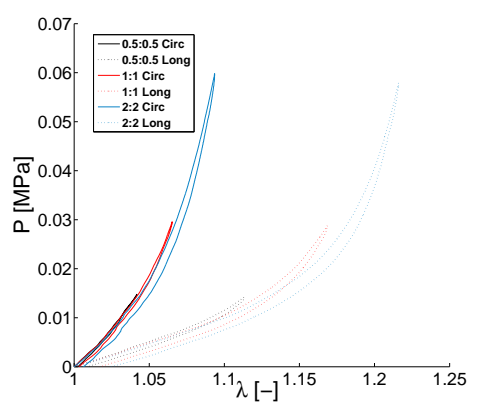

(a) Equibiaxial cycles

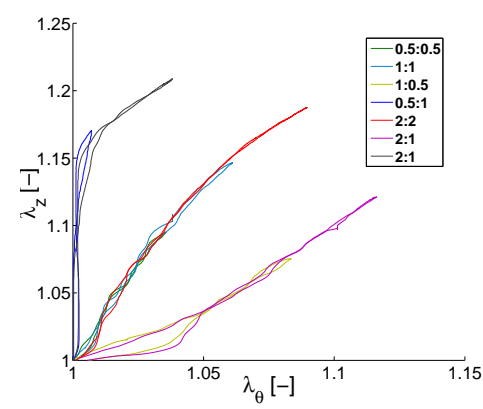

(d) Stretch

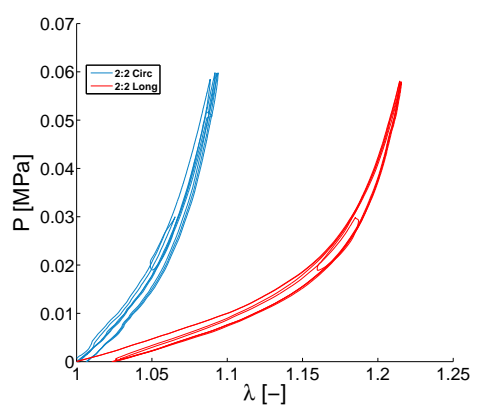

(b) Preconditioning 2:2

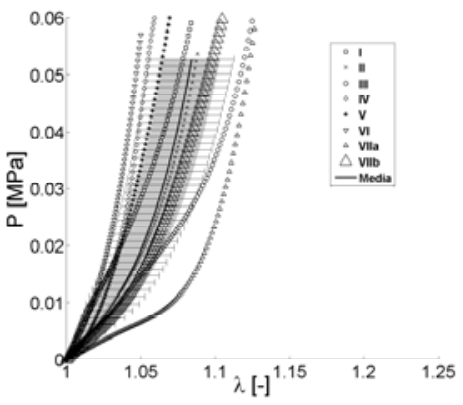

(e) Circumferential

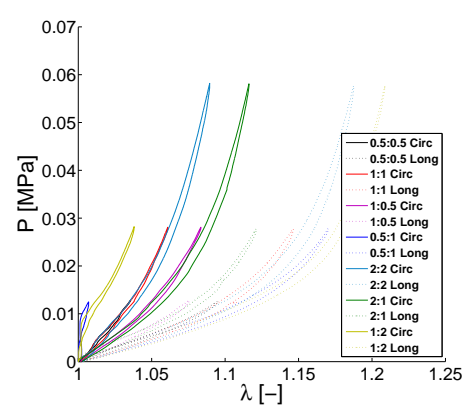

(c) Biaxial

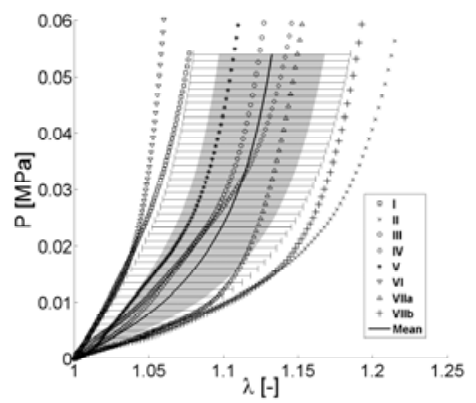

(f) Longitudinal

Fig. 4. Representative (specimen VIIa) Piola-Kirchhoff stress vs. stretch behavior for the porcine descending thoracic aorta (DTA) specimens: (a) typical preconditioned equibiaxial properties at subsequently increased stress levels ranging from 15 to 60 [kPa]; (b) representative preconditioning behavior in terms of loading-unloading cycles at $60[\mathrm{kPa}]$; (c) typical biaxial behavior at different ratios 0.5:0.5, 1:0.5, 0.5:1, 1:1, 2:1, 1:2, $2: 2$; (d) typical axial stretch versus circumferential stretch at different ratios; (e) whole equibiaxial 2:2 experimental data samples in circumferential and (f) longitudinal directions. Colored areas represent $\mathrm{p}>0: 1$ and $\mathrm{p}<0: 9$ of the equibiaxial 2:2 experimental data. 


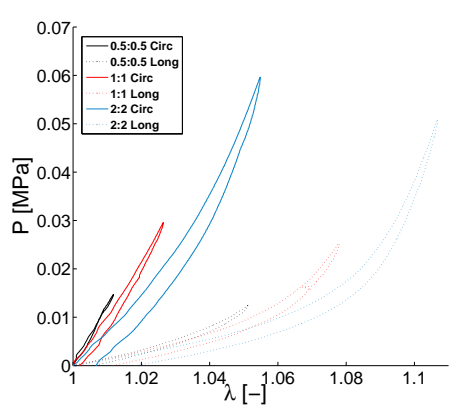

(a) Equibiaxial cycles

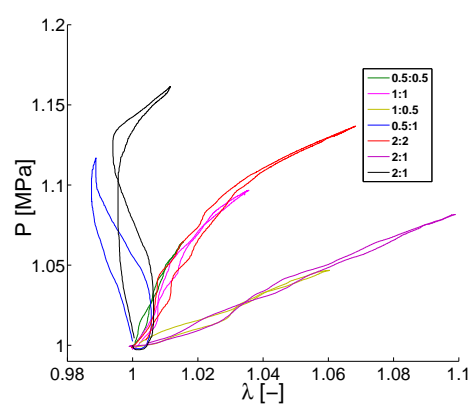

(d) Stretch

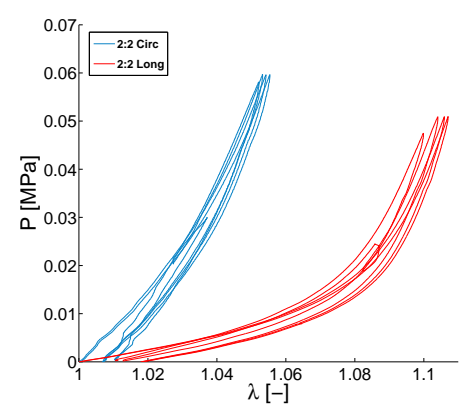

(b) Preconditioning 2:2

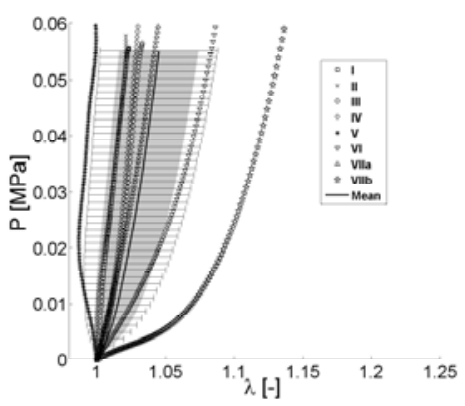

(e) Circumferential

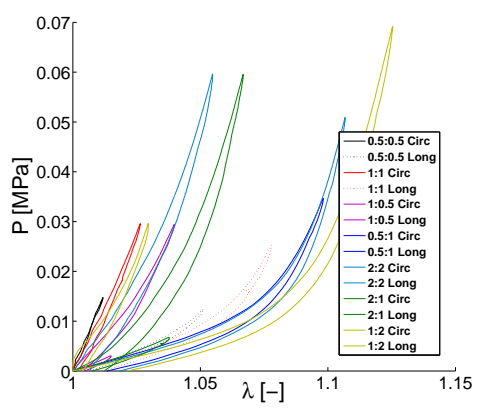

(c) Biaxial

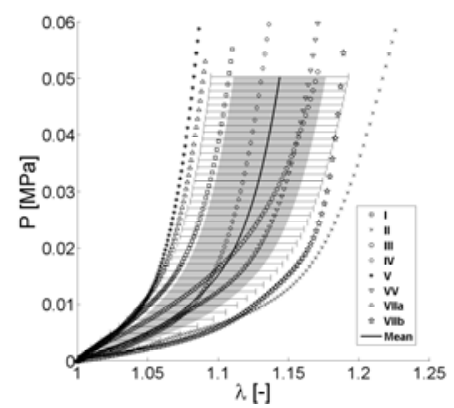

(f) Longitudinal

Fig. 5. Representative (specimen VIIb) Piola-Kirchhoff stress vs. stretch behavior for the distal parts of porcine infrarenal abdominal aorta (IAA) specimens: (a) typical preconditioned equibiaxial properties at subsequently increased stress levels ranging from 15 to $60[\mathrm{kPa}]$; (b) representative preconditioning behavior in terms of loading-unloading cycles at $60[\mathrm{kPa}]$; (c) typical biaxial behavior at different ratios 0.5:0.5, 1:0.5, 0.5:1, 1:1, 2:1, 1:2, 2:2; (d) typical axial stretch versus circumferential stretch at different ratios; (e) whole equibiaxial 2:2 experimental data samples in circumferential and (f) longitudinal directions. Colored areas represent $\mathrm{p}>0: 1$ and $\mathrm{p}<0: 9$ of the equibiaxial 2:2 experimental data. 


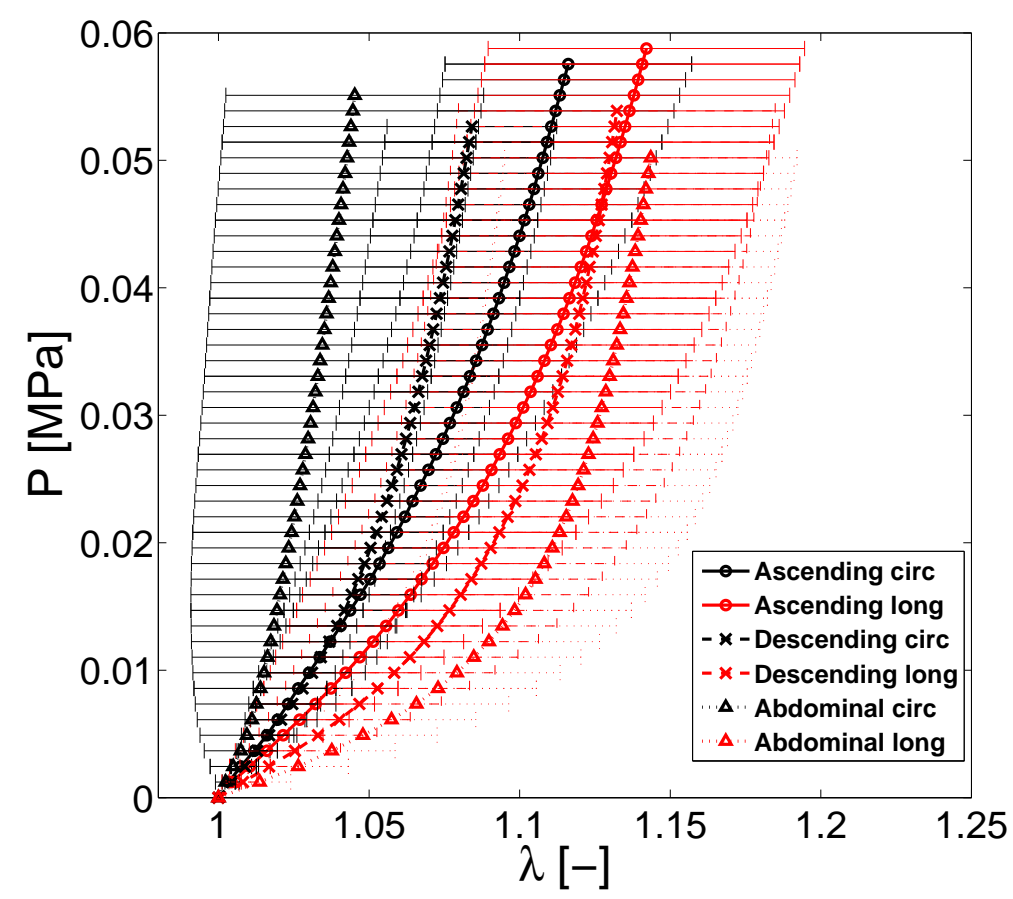

Fig. 6. Average biaxial Cauchy stress-stretch behavior obtained from all considered aortic specimens 